\title{
Activity and different types of electric shock stimuli,'2
}

mLLIAM J. MeCLELLAND AND FRANK D. COLMAN

UNIVERSITY OF WESTERN ONTARIO
Two hundred and sixteen male hooded rats, 140 days old, were shocked in a Campbell and Teghtsoonian learned-fear situation. Aversion thresholds were determined and different groups were exposed to constant voltage $A C$, matched impedance $\mathrm{AC}$, constant current $\mathrm{AC}$, and constant current $\mathrm{DC}$. All sources, excluding constant voltage, caused freezing behavior up to a maximum intensity specific to the source. Results cast doubt on the concept of a single ideal shock for behavioral studies.

The ploneering study of Campbell \& Teghtsoonian (1958) concerning the effects of different shock stimuli has been markedly influential in the design of experiments. Based on a criterion for a "good shock" requiring a proportional increase in a rat's activity for each increment in shock intensity, these authors advocated a constant power source, i.e., a matched impedance source, to prevent the reduction in activity associated with constant current of low intensity. Since recent studies indicate that rats exhibit considerable freezing when shocked by a matched impedance source, it appeared vital to replicate the essential design of the Campbell and Teghtsoonian experiment. Employing the current density concept of Campbell \& Teghtsoonian (1958) and Cornsweet (1963), considerable immobility in animals shocked from constant current ac and dc sources and from the matched impedance ac source was expected, as contrasted to the constant voltage ac stimulation. It was argued that freezing would increase with intensity of the first three sources to an unknown maximum for each source, above which such behavior would decrease. In contrast would be the increase in movement as a function of shock intensity from a constant voltage ac source. Method

The method employed followed as closely as possible that of the Campbell and Teghtsoonian experiment, and the shock sources used (i.e., 60 cycle constant current ac, constant voltage ac, and matched impedance ac; and, constant current dc) were functionally identical. ${ }^{3}$ The modified Miller learned-fear discrimination cage was also similar but, in contrast with the traditional use of the cage, both sides were black to prevent any color discrimination from confounding the discrimination of shock intensity.

Rather than employing the method of independent, discrete readings from the sweep of an oscilloscope used by Campbell and Teghtsoonian, activity or movement of Ss was monitored with a VTVM, as voltage drops over a resistor in series with the rat, and re- corded continuously on a chart recorder. Number of cage tilts was also recorded.

The effects of the four shock sources on activity were compared by utllizing an aversion threshold as a common reference point. Employing the decibel as the unit measure of shock intensity, the actual avoidance threshold (i.e., $0 \mathrm{~dB}$ ) was the intensity of shock which the $\mathrm{S}$ avoided $75 \%$ of the time in the spatial preference situation.

In determining the aversion threshold, 80 male hooded rats, 140 days old, were randomly assigned to groups of 20, each group receiving a specific type of shock. Since a pilot investigation demonstrated that a descending order of shocks resulted in increased freezing behavior, on four successive days each $S$ received one intensity per day for 10 min. in an ascending order. During testing, eight comparable Ss were shocked at each individual decibel level for each shock type at approximately the same intensities used by Campbell and Teghtsoonian. The intensity was raised in decibel levels for each source, to the lowest tetanization level (i.e., the level at which any $S$ of the group became tetanized), or as high as the transformer would allow if tetanization was not reached.

Resulis

The avoidance thresholds of the two studies (Campbell and Teghtsoonian values in brackets) were strikingly similar: constant voltage ac-15 V (10), matched impedance ac-32 V (30), constant current ac-0.10 mA (0.063), and constant current dc- $0.02 \mathrm{~mA}(0.04)$; so were the avoidance functions for the four shock sources.

Analysis of variance of the amount of freezing by shock source revealed significant source effects ( $F=$ 74.2, df $=3 / 84, p<.001$ ). The constant current dc source elicited significantly more freezing behavior $(p<.001)$. The analysis of freezing by decibel levels was significant $(F=3.1$, df $=2 / 84, p<.05)$ as were the interactions of decibel level by trial $(F=8.0$, $\mathrm{df}=2 / 84, \mathrm{p}<.001)$, and source by trial $(F=7.9, \mathrm{df}=$ $3 / 84, \mathrm{p}<.001$ ).

The analysis of variance of number of cage tilts by decibel level was significant $(F=59.6, \mathrm{df}=2 / 84$, $\mathrm{p}<.001$ ), though this was due to a single significant difference between the $10 \mathrm{~dB}$ and the other two levels $(p<.001)$. The analysis of the effects of different shock types was also significant $(F=30.5$, $\mathrm{df}=3 / 84$, $p<.001)$. The number of tilts decreased significantly from test trial 1 to test trial $2(F=300.8, d f=1 / 84$, $p<.001)$. Interactions of decibel level by type of 


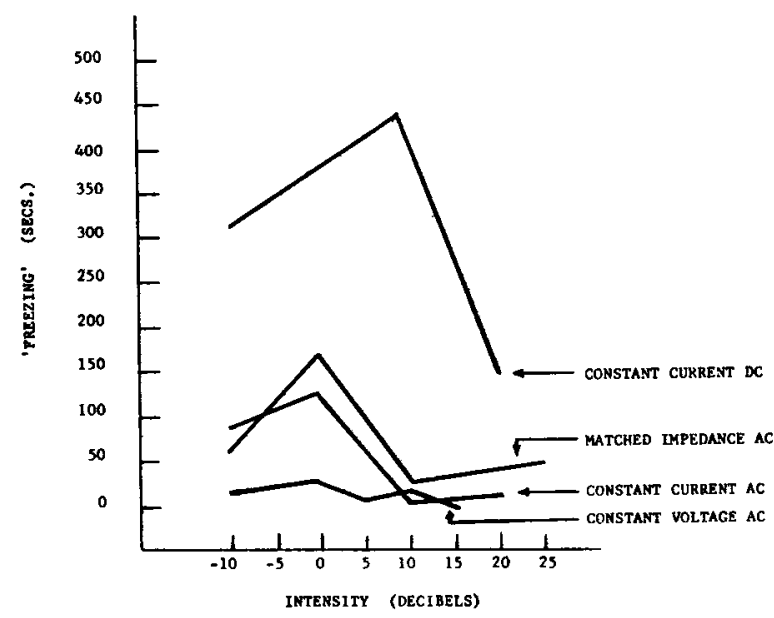

Fig. 1. The effect of intensity and shock type on freezing behavior.

shock $(F=5.8, d f=6 / 84, p<.01)$; trials by decibel level $(F=126.2, d f=2 / 84, p<.001)$; trials by source $(F=16.5, d f=6 / 84, p<.001)$; and trials by decibel level by type of shock $(F=11.0, d f=6 / 84, p<.001)$ were significant.

For both matched impedance ac and constant current ac, amount of freezing increased to the $0 \mathrm{~dB}$ level and then decreased sharply. As one might expect, as the freezing decreased, the number of cage tilts increased significantly. The constant voltage source elicited the least amount of freezing at all intensities, and the number of cage tilts increased significantly at the $10 \mathrm{~dB}$ level for this source. Constant current dc elicited the greatest amount of freezing at all intensities, decreasing markedly at the $20 \mathrm{~dB}$ level with, of course, a corresponding significant increase of cage tilts.

\section{Discussion}

The data supports the predictions based on the current density arguments. Rats receiving electric shock from constant current ac, constant current dc, and matched impedance ac sources, responded by increasing freezing as intensity increased up to a given level for each source. Rats receiving shock from these same sources, greater than this individual maximum intensity, responded by increased activity. And finally, rats receiving electric shock from a constant voltage ac source exhibited very little freezing behavior but instead responded by increasing random movement as the Intensity increased.

The reason for considerable freezing from the constant current dc source may be the pulse of current each time the $S$ makes or breaks contact with the grid. Freezing behavior is quite reinforcing when the rat is receiving constant current dc shock, since remaining still would eliminate this pulse. The constant voltage shock source produced the least amount of freezing behavior. This result was predicted on the basis of Cornsweet's argument that the constant voltage circuit tends to give a more constant current density than the constant current circuit. Accordingly, a rat in a constant current shock situation would learn to crouch and freeze (since this reduces the current density) as well as not jump from the grids (since at both the instant of leaving the grid and recontacting it, the areas of contact will be very small and the current density very great). A rat receiving constant voltage shock might learn the opposite pattern.

The matched impedance ac shock source produced a significantly greater amount of freezing than the constant voltage source, an amount that was not significantly different but greater than that elicited by the constant current ac source. The close similarity of the behavioral patterns elicited by the matched impedance and constant current ac sources indicates that a matched impedance source is much closer functionally to a constant current ac source as was expected. However, at highest levels this latter source approaches constant voltage and hence would result in movement responses once more.

The results suggest that there is no single ideal shock for behavioral studies such as the matched impedance source typically employed. Instead of one ideal shock, the type to be used depends entirely on the responses desired. If activity or movement responses are of interest, then a low intensity constant voltage ac shock source should be employed. If freezing is the response being studied, then a constant current dc source at low and medium intensities is suitable.

\section{Reterences}

Campbell, B. A., \& Teghtsoonian, R. Electrical and behavioral effects of different types of shock stimuli on the rat. $J$. comp. physiol. Psychol., 1958, 51, 185-192.

Comsweet, T. N. The design of electrical circuits in the behavioral sciences. New York: Wiley \& Sons, 1963.

\section{Notes}

1. This investigation was supported by Grant No. 30 from the Ontario Mental Health Foundation to the senior author.

2. Thanks are due to J. T. Hawkins for his assistance with this study.

3. Schematic diagrams of the $A C$ and $D C$ shock circuits are available on request. 